Mathematical Modelling and AnAlysis

Volume 21 Number 2, March 2016, 260-269

http://dx.doi.org/10.3846/13926292.2016.1150904

(c) Vilnius Gediminas Technical University, 2016
Publisher: Taylor\&Francis and VGTU

http://www.tandfonline.com/TMMA

ISSN: $1392-6292$

eISSN: $1648-3510$

\title{
Fujita Critical Curve for a Coupled Diffusion System with Inhomogeneous Neumann Boundary Conditions*
}

\section{Runmei Du and Minghao Guo}

Changchun University of Technology

School of Basic Science, 130012 Changchun, China

E-mail(corresp.): mhguo@ccut.edu.cn

E-mail: durm_dudu@163.com

Received September 23, 2015; revised February 1, 2016; published online March 15, 2016

\begin{abstract}
In this paper, we establish the blow-up theorems of Fujita type for a class of exterior problems of nonlinear diffusion equations subject to inhomogeneous Neumann boundary conditions. The critical Fujita exponents are determined and it is shown that the critical curve belongs to the blow-up case under any nontrivial initial data.
\end{abstract}

Keywords: Fujita critical curve, inhomogeneous term, global existence, blow-up, diffusion equations.

AMS Subject Classification: 35K65; 35B33.

\section{Introduction}

In this paper, we consider the critical curve of the following coupled diffusion system with inhomogeneous Neumann boundary conditions

$$
\begin{cases}u_{t}=\Delta u^{m}+v^{p}, \quad v_{t}=\Delta v^{n}+u^{q}, & (x, t) \in D^{c} \times(0,+\infty), \\ \frac{\partial u^{m}}{\partial \nu}(x, t)=f_{1}(x), \quad \frac{\partial v^{n}}{\partial \nu}(x, t)=f_{2}(x), & (x, t) \in \partial D \times(0,+\infty), \\ u(x, 0)=u_{0}(x), \quad v(x, 0)=v_{0}(x), & x \in D^{c}\end{cases}
$$

where $m, n>0, p>\max \{1, n\}, q>\max \{1, m\}, D$ is a bounded open domain in $\mathbb{R}^{N}$ with smooth boundary, $D^{c}=\mathbb{R}^{N} \backslash \bar{D}, \nu$ is the inward unit normal to $\partial D$. $u_{0}(x), v_{0}(x)$ are nonnegative continuous functions in $D^{c}, f_{1}(x), f_{2}(x)$ are nontrivial nonnegative continuous functions on $\partial D$, and $u_{0}(x)=f_{1}(x), v_{0}(x)=$ $f_{2}(x)$ on $\partial D$.

\footnotetext{
* Supported by National Natural Science Foundation of China (Grants No. 11401049).
} 
Study on critical exponents began from 1966 by Fujita in the work [6]. He considered the Cauchy problem

$$
\begin{cases}u_{t}=\Delta u+u^{p}, & x \in \mathbb{R}^{N}, t>0, \\ u(x, 0)=u_{0}(x), & x \in \mathbb{R}^{N}\end{cases}
$$

where $u_{0} \geq 0$. It was shown that the problem has no non-trivial global solutions if $1<p<p_{c}=1+2 / N$, whereas if $p>p_{c}$, there exist both global (with small initial data) and non-global (with large initial data) solutions. We call $p_{c}$ the critical Fujita exponent. Later, it was proved by Hayakawa [7] and Weissler [10] that $p=p_{c}$ belongs to the blow-up case. From then on, there have been a lot of works on the critical exponents of Fujita type for various nonlinear evolution equations and systems [1-18]. Among these, Zeng [12] investigated the blow-up theorems of Fujita type for the following inhomogeneous problems

$$
\begin{cases}u_{t}=\Delta u^{m}+u^{p}+f(x), & x \in \mathbb{R}^{N}, t>0, \\ u(x, 0)=u_{0}(x), & x \in \mathbb{R}^{N},\end{cases}
$$

where $m>0, p>\max \{1, m\}, f(x)$ and $u_{0}(x)$ are nonnegative continuous functions in $\mathbb{R}^{N}$ with $f(x) \not \equiv 0$, and

$$
\begin{cases}u_{t}=\Delta u^{m}+u^{p} & (x, t) \in D^{c} \times(0,+\infty), \\ \frac{\partial u^{m}}{\partial \nu}(x, t)=f(x), & (x, t) \in \partial D \times(0,+\infty), \\ u(x, 0)=u_{0}(x), & x \in D^{c},\end{cases}
$$

where $m>0, p>\max \{1, m\}, f(x)$ is a nontrivial nonnegative continuous function on $\partial D, u_{0}(x)$ is a nonnegative continuous function in $D^{c}$. The author proved that $p_{c}=m+2 m /(N-2)_{+}$is the critical exponent of both the problems.

As for coupled systems, Escobedo and Herrero [5] studied the Cauchy problem

$$
\begin{cases}u_{t}=\Delta u+v^{p}, \quad v_{t}=\Delta v+u^{q}, & x \in \mathbb{R}^{N}, t>0, \\ u(x, 0)=u_{0}(x), \quad v(x, 0)=v_{0}(x), & x \in \mathbb{R}^{N}\end{cases}
$$

They proved that the critical Fujita curve is $(p q)_{c}=1+\frac{2}{N} \max \{p+1, q+1\}$, namely, every solution blows up in finite time if $1<p q \leq(p q)_{c}$, and there exist both global and non-global solutions if $p q>(p q)_{c}$.

Furthermore, Yang et al. [11] studied the fast diffusion system

$$
\begin{cases}u_{t}=\Delta u^{m}+v^{p}+f_{1}(x), \quad v_{t}=\Delta v^{n}+u^{q}+f_{2}(x), & x \in \mathbb{R}^{N}, t>0, \\ u(x, 0)=u_{0}(x), \quad v(x, 0)=v_{0}(x), & x \in \mathbb{R}^{N},\end{cases}
$$

where $0<m, n<1, p, q \geq 1, p q>1$, and determined the critical curve

$$
(p q)_{c}=m n+\frac{2}{(N-2)_{+}} \max \{m p+m n, n q+m n\} .
$$

The aim of this paper is to determine the critical curve to the coupled diffusion system (1.1). In the present paper, we prove the critical Fujita curve 
for the system (1.1) is also

$$
(p q)_{c}=m n+\frac{2}{(N-2)_{+}} \max \{m p+m n, n q+m n\} .
$$

Namely, every solution blows up in finite time if $p q<(p q)_{c}$, and there exist both global and non-global solutions if $p q>(p q)_{c}$. Further, we prove that the case $p q=(p q)_{c}$ belongs to the blow-up case. The method we used is similar to $[1,2,3,9,11,12]$.

\section{Main results and their proofs}

First, we give the definition of the solution to the problem (1.1).

Definition 1. A pair of functions $(u, v)$ is said to be a weak solution of the problem (1.1), if $u \in C\left(0, T ; L_{l o c}^{1}\left(D^{c}\right)\right) \cap C\left(0, T ; L_{l o c}^{q}\left(D^{c}\right)\right), v \in C\left(0, T ; L_{l o c}^{1}\left(D^{c}\right)\right)$ $\cap C\left(0, T ; L_{l o c}^{p}\left(D^{c}\right)\right), \nabla u^{m}, \nabla v^{n} \in L^{2}\left(0, T ; L_{l o c}^{2}\left(D^{c}\right)\right)$ and satisfy

$$
\begin{aligned}
\int_{0}^{\tau} \int_{D^{c}}\left(u \frac{\partial \psi_{1}}{\partial t}-\nabla u^{m} \nabla \psi_{1}+v^{p} \psi_{1}\right) & d x d t+\int_{0}^{\tau} \int_{\partial D} f_{1} \psi_{1} d S d t \\
& =\int_{D^{c}} u \psi_{1}(x, \tau)-\int_{D^{c}} u_{0}(x) \psi_{1}(x, 0) d x \\
\int_{0}^{\tau} \int_{D^{c}}\left(v \frac{\partial \psi_{2}}{\partial t}-\nabla v^{n} \nabla \psi_{2}+u^{q} \psi_{2}\right) & d x d t+\int_{0}^{\tau} \int_{\partial D} f_{2} \psi_{2} d S d t \\
& =\int_{D^{c}} v \psi_{2}(x, \tau)-\int_{D^{c}} v_{0}(x) \psi_{2}(x, 0) d x
\end{aligned}
$$

for any $\tau \in[0, T]$ and any compactly supported $\psi_{1}, \psi_{2} \in C^{2}\left(D^{c} \times[0, T]\right) \cap C\left(\overline{D^{c}} \times\right.$ $[0, T])$.

The standard theory of parabolic equations ensures the well-posedness of the problem (1.1). Furthermore, we introduce the comparison principle $([4,8,13])$ and the monotonicity property( [12]) of the system (1.1) and omit the proof.

Lemma 1. Let $u_{1}, u_{2} \in C\left(0, T ; L_{l o c}^{1}\left(D^{c}\right)\right) \cap C\left(0, T ; L_{l o c}^{q}\left(D^{c}\right)\right), v_{1}, v_{2} \in C(0, T$; $\left.L_{l o c}^{1}\left(D^{c}\right)\right) \cap C\left(0, T ; L_{l o c}^{p}\left(D^{c}\right)\right), \nabla u_{1}^{m}, \nabla u_{2}^{m}, \nabla v_{1}^{n}, \nabla v_{2}^{n} \in L^{2}\left(0, T ; L_{l o c}^{2}\left(D^{c}\right)\right)$ and satisfy

$$
\begin{array}{ll}
\left(u_{1}\right)_{t}-\Delta u_{1}^{m}-v_{1}^{p} \leq\left(u_{2}\right)_{t}-\Delta u_{2}^{m}-v_{2}^{p}, & (x, t) \in D^{c} \times(0,+\infty), \\
\left(v_{1}\right)_{t}-\Delta v_{1}^{n}-u_{1}^{q} \leq\left(v_{2}\right)_{t}-\Delta v_{2}^{n}-u_{2}^{q}, & (x, t) \in D^{c} \times(0,+\infty), \\
\frac{\partial u_{1}^{m}}{\partial \nu}(x, t) \leq \frac{\partial u_{2}^{m}}{\partial \nu}(x, t), \quad \frac{\partial v_{1}^{n}}{\partial \nu}(x, t) \leq \frac{\partial v_{2}^{n}}{\partial \nu}(x, t), & (x, t) \in \partial D \times(0,+\infty), \\
u_{1}(x, 0) \leq u_{2}(x, 0), \quad v_{1}(x, 0) \leq v_{2}(x, 0), & x \in D^{c},
\end{array}
$$

then

$$
u_{1}(x, t) \leq u_{2}(x, t), \quad v_{1}(x, t) \leq v_{2}(x, t), \quad(x, t) \in D^{c} \times(0,+\infty)
$$


Lemma 2. The nonnegative solutions of (1.1) with zero initial data must be monotone increasing to $t$ for both components $u$ and $v$.

Now, we prove that the Fujita critical curve is

$$
(p q)_{c}=m n+\frac{2}{(N-2)_{+}} \max \{m p+m n, n q+m n\} .
$$

The main results are as follows.

Theorem 1. If $p q \leq(p q)_{c}$, then the system (1.1) has no nontrivial nonnegative global solution.

Proof. Without loss of generality, assume $m p \geq n q$. Let $R_{0}>1$ be a fixed constant such that $D \subset B_{R_{0} / 2}(0)$. For $R>R_{0}, T>1$, choose two smooth functions $\psi_{R}(x)$ and $\eta_{T}(t)$ satisfying:

$$
\begin{aligned}
& \psi_{R}(x)=1, \quad 0 \leq|x| \leq R / 2 ; \quad \psi_{R}(x)=0, \quad|x|>R, \quad 0 \leq \psi_{R}(x) \leq 1, \quad|x|>0 \\
& \left|\frac{\partial \psi_{R}}{\partial x_{i}}(x)\right| \leq \frac{C}{R}, \quad\left|\frac{\partial^{2} \psi_{R}}{\partial x_{i} \partial x_{j}}(x)\right| \leq \frac{C}{R^{2}}, \quad i, j=1,2, \cdots, N ; \\
& \eta_{T}(t)=1, \quad 0 \leq t \leq T / 2 ; \quad \eta_{T}(t)=0, t>T, \quad 0 \leq \eta_{T}(t) \leq 1, t>0 ; \\
& \left|\eta_{T}^{\prime}(t)\right| \leq \frac{C}{T}, \quad\left|\eta_{T}^{\prime \prime}(t)\right| \leq \frac{C}{T^{2}} .
\end{aligned}
$$

Here and in the sequels, we use $C$ to represent positive constants independent of $R$ and $T$. Suppose by contradiction that $(u, v)$ is a nontrivial global solution of (1.1). Define

$$
\begin{aligned}
I_{p} & =\int_{0}^{T} \int_{B_{R}(0) \backslash \bar{D}} v^{p}(x, t) \psi_{R}^{l}(x) \eta_{T}^{l}(t) d x d t, \\
J_{q} & =\int_{0}^{T} \int_{B_{R}(0) \backslash \bar{D}} u^{q}(x, t) \psi_{R}^{l}(x) \eta_{T}^{l}(t) d x d t,
\end{aligned}
$$

where $l>\max \{p /(p-1), q /(q-1)\}$. Noting the direction of $\nu$ and $\eta_{T}(T)=0$, we have

$$
\begin{aligned}
I_{p}= & \int_{0}^{T} \int_{\mathbb{R}^{N} \backslash \bar{D}}\left(u_{t}(x, t)-\Delta u^{m}(x, t)\right) \psi_{R}^{l}(x) \eta_{T}^{l}(t) d x d t \\
= & {\left[\int_{\mathbb{R}^{N} \backslash \bar{D}} u(x, t) \psi_{R}^{l}(x) \eta_{T}^{l}(t) d x\right]_{0}^{T}-\int_{0}^{T} \int_{\mathbb{R}^{N}} u(x, t) \psi_{R}^{l}(x)\left(\eta_{T}^{l}(t)\right)^{\prime} d x d t } \\
& -\int_{0}^{T} \int_{\mathbb{R}^{N} \backslash \bar{D}} u^{m}(x, t) \Delta\left(\psi_{R}^{l}(x)\right) \eta_{T}^{l}(t) d x d t-\int_{0}^{T} \int_{\partial D} f_{1}(x) \eta_{T}^{l}(t) d x d t \\
= & -\int_{B_{R}(0) \backslash \bar{D}} u_{0}(x) \psi_{R}^{l}(x) d x-\int_{0}^{T} \int_{\mathbb{R}^{N}} u(x, t) \psi_{R}^{l}(x)\left(\eta_{T}^{l}(t)\right)^{\prime} d x d t \\
& -\int_{0}^{T} \int_{\mathbb{R}^{N} \backslash \bar{D}} u^{m}(x, t) \Delta\left(\psi_{R}^{l}(x)\right) \eta_{T}^{l}(t) d x d t-\int_{0}^{T} \int_{\partial D} f_{1}(x) \eta_{T}^{l}(t) d x d t .
\end{aligned}
$$


Since for any $i=1,2, \cdots, N$,

$$
\frac{\partial^{2} \psi_{R}^{l}(x)}{\partial x_{i}^{2}}=\frac{\partial}{\partial x_{i}}\left(l \psi_{R}^{l-1} \frac{\partial \psi_{R}(x)}{\partial x_{i}}\right)=l \psi_{R}^{l-1} \frac{\partial^{2} \psi_{R}(x)}{\partial x_{i}^{2}}+l(l-1) \psi_{R}^{l-2}\left(\frac{\partial \psi_{R}(x)}{\partial x_{i}}\right)^{2},
$$

it holds

$$
\Delta \psi_{R}^{l}(x) \geq l \psi_{R}^{l-1}(x) \sum_{i=1}^{N} \frac{\partial^{2} \psi_{R}(x)}{\partial x_{i}^{2}}
$$

Then,

$$
\begin{aligned}
I_{p} & \leq-\int_{B_{R}(0) \backslash \bar{D}} u_{0}(x) \psi_{R}^{l}(x) d x+C T^{-1} \int_{T / 2}^{T} \int_{B_{R}(0) \backslash} u(x, t) \psi_{R}^{l}(x) \eta_{T}^{l-1}(t) d x d t \\
& +C R^{-2} \int_{0}^{T} \int_{B_{R}(0) \backslash B_{R / 2}(0)} u^{m}(x, t) \psi_{R}^{l-1}(x) \eta_{T}^{l}(t) d x d t-\int_{0}^{T} \int_{\partial D} f_{1}(x) \eta_{T}^{l}(t) d x d t .
\end{aligned}
$$

Using Hölder's inequality and noting $l>\max \{p /(p-1), q /(q-1)\}$, we have

$$
\begin{aligned}
I_{p} & \leq-\int_{B_{R}(0) \backslash \bar{D}} u_{0}(x) \psi_{R}^{l}(x) d x+C T^{-1} J_{q}^{1 / q}\left(\int_{T / 2}^{T} \int_{B_{R}(0) \backslash \bar{D}} d x d t\right)^{1-1 / q} \\
& +C R^{-2} J_{q}^{m / q}\left(\int_{T / 2}^{T} \int_{B_{R}(0) \backslash B_{R / 2}(0)} d x d t\right)^{1-m / q}-\int_{0}^{T} \int_{\partial D} f_{1}(x) \eta_{T}^{l}(t) d x d t .
\end{aligned}
$$

Denote

$$
\delta=\min \left\{\int_{\partial D} f_{1}(x) d x, \int_{\partial D} f_{2}(x) d x\right\}
$$

Then

$$
\int_{0}^{T} \int_{\partial D} f_{i}(x) \eta_{T}^{l}(t) d x d t \geq \int_{0}^{T / 2} \int_{\partial D} f_{i}(x) d x d t \geq \frac{\delta}{2} T, \quad i=1,2 .
$$

Together with (2.2), we have

$$
\begin{gathered}
I_{p}+\int_{B_{R}(0) \backslash \bar{D}} u_{0}(x) \psi_{R}^{l}(x) d x+\frac{\delta}{2} T \leq C T^{-1 / q} R^{N(q-1) / q} J_{q}^{1 / q} \\
+C T^{1-m / q} R^{N(q-m) / q-2} J_{q}^{m / q} .
\end{gathered}
$$

Similarly,

$$
\begin{aligned}
& J_{q}+\int_{B_{R}(0) \backslash \bar{D}} v_{0}(x) \psi_{R}^{l}(x) d x+\frac{\delta}{2} T \leq \\
& C T^{-1 / p} R^{N(p-1) / p} I_{p}^{1 / p}+C T^{1-n / p} R^{N(p-n) / p-2} I_{p}^{n / p} .
\end{aligned}
$$


Consequently, we can obtain

$$
\begin{aligned}
I_{p}+ & \int_{B_{R}(0) \backslash \bar{D}} u_{0}(x) \psi_{R}^{l}(x) d x+\frac{\delta}{2} T \leq C T^{-1 / q} R^{N(q-1) / q} \\
& \times\left(C T^{-1 / p} R^{N(p-1) / p} I_{p}^{1 / p}+C T^{1-n / p} R^{N(p-n) / p-2} I_{p}^{n / p}\right)^{1 / q}+C T^{1-m / q} \\
& \times R^{N(q-m) / q-2}\left(C T^{-1 / p} R^{N(p-1) / p} I_{p}^{1 / p}+C T^{1-n / p} R^{N(p-n) / p-2} I_{p}^{n / p}\right)^{m / q} \\
\leq & C T^{-(p+1) /(p q)} R^{N(p q-1) /(p q)} I_{p}^{1 /(p q)}+C T^{-n /(p q)} R^{N(p q-n) /(p q)-2 / q} I_{p}^{n /(p q)} \\
& +C T^{(p q-m p-m) /(p q)} R^{N(p q-m) /(p q)-2} I_{p}^{m /(p q)} \\
& +C T^{(p q-m n) /(p q)} R^{N(p q-m n) /(p q)-2 m / q-2} I_{p}^{m n /(p q)}
\end{aligned}
$$

As $p>\max \{1, n\}, q>\max \{1, m\}$, by Young's inequality, we have

$$
\begin{aligned}
& I_{p}+\int_{B_{R}(0) \backslash \bar{D}} u_{0}(x) \psi_{R}^{l}(x) d x+\frac{\delta}{2} T \leq \frac{1}{8} I_{p}+C T^{-(p+1) /(p q-1)} R^{N}+\frac{1}{8} I_{p} \\
& +C T^{-n /(p q-n)} R^{N-2 p /(p q-n)}+\frac{1}{8} I_{p}+C T^{(p q-m p-m) /(p q-m)} R^{N-2 p q /(p q-m)} \\
& +\frac{1}{8} I_{p}+C T R^{N-2(m p+p q) /(p q-m n)} .
\end{aligned}
$$

Hence,

$$
\begin{aligned}
\frac{\delta}{2} \leq & C T^{-(p+1) /(p q-1)-1} R^{N}+C T^{-n /(p q-n)-1} R^{N-2 p /(p q-n)} \\
& +C T^{-m p /(p q-m)} R^{N-2 p q /(p q-m)}+C R^{N-2(m p+p q) /(p q-m n)} .
\end{aligned}
$$

If $p q<(p q)_{c}$, then $N-\frac{2 p(q+m)}{p q-m n}<0$. Let $R$ be such that $C R^{N-\frac{2 p(q+m)}{p q-m n}}<\delta / 4$. For the fixed $R$, we can choose $T$ such that

$$
\begin{aligned}
& C T^{-(p+1) /(p q-1)-1} R^{N}+C T^{-n /(p q-n)-1} R^{N-2 p /(p q-n)} \\
& \quad+C T^{-m p /(p q-m)} R^{N-2 p q /(p q-m)}<\frac{\delta}{4},
\end{aligned}
$$

leading to a contradiction.

If $p q=(p q)_{c}$, then $N-\frac{2 p(q+m)}{p q-m n}=0$. From Lemma 1, it suffices to prove the blow-up of $(u, v)$ with $u_{0}=v_{0}=0$. By Lemma $2, u_{t}, v_{t} \geq 0$. Choose $T>0$ large such that the sum of first three terms in right hand side of (2.7) is smaller than 1. It follows from (2.6) that for fixed $R>R_{0}$,

$$
\int_{\frac{T}{4}}^{\frac{T}{2}} \int_{B_{R / 2}} v^{p}(x, t) d x d t \leq C T
$$

and consequently,

$$
\inf _{t \in\left(\frac{T}{4}, \frac{T}{2}\right)} \int_{B_{R / 2}} v^{p}(x, t) d x d t \leq C .
$$


Due to $v_{t} \geq 0$ and the arbitrary of $T$, we have $\int_{B_{R / 2}} v^{p}(x, t) d x \leq C$ for $t>0$ and hence

$$
I_{R}^{\infty}:=\lim _{t \rightarrow \infty} \int_{B_{\frac{R}{2}}} v^{p}(x, t) d x \leq C .
$$

Since $I_{R}^{\infty}$ is increasing with $R, \lim _{R \rightarrow \infty} I_{R}^{\infty}$ exists. So, for any $\varepsilon>0$, there exists $R_{1}$, such that for $R \geq R_{1}$,

$$
\int_{B_{R}(0) \backslash B_{R / 2}(0)} v^{p}(x, t) d x<\varepsilon, \quad t>0 .
$$

Similar to (2.1), we get

$$
\begin{aligned}
& J_{q} \leq-\int_{B_{R}(0) \backslash \bar{D}} v_{0}(x) \psi_{R}^{l}(x) d x+C T^{-1} \int_{T / 2}^{T} \int_{B_{R}(0) \backslash \bar{D}} v(x, t) \psi_{R}^{l}(x) \eta_{T}^{l-1}(t) d x d t \\
& +C R^{-2} \int_{0}^{T} \int_{B_{R}(0) \backslash B_{R / 2}(0)} v^{n}(x, t) \psi_{R}^{l-1}(x) \eta_{T}^{l}(t) d x d t-\int_{0}^{T} \int_{\partial D} f_{2}(x) \eta_{T}^{l}(t) d x d t .
\end{aligned}
$$

By Hölder inequality, (2.3) and (2.9), we have

$$
\begin{aligned}
J_{q} & +\int_{B_{R}(0) \backslash \bar{D}} v_{0}(x) \psi_{R}^{l}(x) d x+\frac{\delta}{2} T \leq C T^{-1 / p} R^{N(p-1) / p} I_{p}^{1 / p} \\
& +C T^{1-n / p} R^{N(p-n) / p-2}\left(\int_{0}^{T} \int_{B_{R}(0) \backslash B_{R / 2}(0)} v^{p} \psi_{R}^{l}(x) \eta_{T}^{l}(t) d x d t\right)^{n / p} \\
& \leq C T^{-1 / p} R^{N(p-1) / p} I_{p}^{1 / p}+C T R^{N(p-n) / p-2} \varepsilon^{n / p}
\end{aligned}
$$

From $(2.4),(2.5),(2.10)$ and $p q=(p q)_{c}$, we obtain

$$
\begin{aligned}
& I_{p}+\int_{B_{R}(0) \backslash \bar{D}} u_{0}(x) \psi_{R}^{l}(x) d x+\frac{\delta}{2} T \\
& \leq C T^{-1 / q} R^{N(q-1) / q}\left(C T^{-1 / p} R^{N(p-1) / p} I_{p}^{1 / p}+C T^{1-n / p} R^{N(p-n) / p-2} I_{p}^{n / p}\right)^{1 / q} \\
& +C T^{1-m / q} R^{N(q-m) / q-2}\left(C T^{-1 / p} R^{N(p-1) / p} I_{p}^{1 / p}+C T R^{N(p-n) / p-2} \varepsilon^{n / p}\right)^{m / q} \\
& \leq C T^{-(p+1) /(p q)} R^{N(p q-1) /(p q)} I_{p}^{1 /(p q)}+C T^{-n /(p q)} R^{N(p q-n) /(p q)-2 / q} I_{p}^{n /(p q)} \\
& +C T^{(p q-m p-m) /(p q)} R^{N(p q-m) /(p q)-2} I_{p}^{m /(p q)}+C T \varepsilon^{m n /(p q)} .
\end{aligned}
$$

By Young inequality, we have

$$
\begin{aligned}
I_{p}+ & \delta T \leq \frac{1}{6} I_{p}+C T^{-(p+1) /(p q-1)} R^{N}+\frac{1}{6} I_{p}+C T^{-n /(p q-n)} R^{N-2 p /(p q-n)} \\
& +\frac{1}{6} I_{p}+C T^{(p q-m p-m) /(p q-m)} R^{N-2 p q /(p q-m)}+C T \varepsilon^{m n /(p q)},
\end{aligned}
$$

which implies

$$
\begin{aligned}
\frac{\delta}{2} \leq & C T^{-(p+1) /(p q-1)-1} R^{N}+C T^{-n /(p q-n)-1} R^{N-2 p /(p q-n)} \\
& +C T^{-m p /(p q-m)} R^{N-2 p q /(p q-m)}+C \varepsilon^{m n /(p q)} .
\end{aligned}
$$


Take $\varepsilon<\left(\frac{\delta}{4 C}\right)^{p q /(m n)}$, there exists $R_{2}$, such that for a fixed $R>R_{2},(2.9)$ is valid. Then choose $T$ large enough such that (2.8) holds, a contradiction.

Theorem 2. If $p q>(p q)_{c}$, then there exist solutions of the system (1.1) blow up in finite time.

Proof. Suppose by contradiction that every solution $(u, v)$ of the system (1.1) is a global solution. Similar to the proof of Theorem 1 , we assume $m p \geq n q$. Then, for any fixed $R>R_{0}, T>1$, we have (2.6). Thus,

$$
\begin{aligned}
& \int_{B_{R / 2}(0) \backslash B_{R_{0} / 2}(0)} u_{0}(x) d x \\
\leq & C T^{-(p+1) /(p q-1)} R^{N}+C T^{-n /(p q-n)} R^{N-2 p /(p q-n)} \\
& +C T^{(p q-m p-m) /(p q-m)} R^{N-2 p q /(p q-m)}+C T R^{N-2(m p+p q) /(p q-m n)} .
\end{aligned}
$$

Let $\kappa$ be large enough such that

$$
\begin{aligned}
& \kappa \int_{B_{R / 2}(0) \backslash B_{R_{0} / 2}(0)} 1 d x \\
> & C T^{-(p+1) /(p q-1)} R^{N}+C T^{-n /(p q-n)} R^{N-2 p /(p q-n)} \\
& +C T^{(p q-m p-m) /(p q-m)} R^{N-2 p q /(p q-m)}+C T R^{N-2(m p+p q) /(p q-m n)} .
\end{aligned}
$$

Then for $u_{0}(x)>\kappa \psi_{R}(x)$, we have

$$
\begin{aligned}
& \int_{B_{R / 2}(0) \backslash B_{R_{0} / 2}(0)} u_{0}(x) d x>\kappa \int_{B_{R / 2}(0) \backslash B_{R_{0} / 2}(0)} 1 d x \\
> & C T^{-(p+1) /(p q-1)} R^{N}+C T^{-n /(p q-n)} R^{N-2 p /(p q-n)} \\
& +C T^{(p q-m p-m) /(p q-m)} R^{N-2 p q /(p q-m)}+C T R^{N-2(m p+p q) /(p q-m n)},
\end{aligned}
$$

which contradicts $(2.11)$.

Theorem 3. If $p q>(p q)_{c}$, then there exist global solutions of the system (1.1).

Proof. Note $p q>(p q)_{c}$ implies $N-\max \left\{\alpha_{1} m+2, \alpha_{2} n+2\right\}>0$. Define

$$
\begin{aligned}
& M_{1}=\left(\alpha_{1} m\left(N-\alpha_{1} m-2\right)\right)^{n /(p q-m n)}\left(\alpha_{2} n\left(N-\alpha_{2} n-2\right)\right)^{p /(p q-m n)}, \\
& M_{2}=\left(\alpha_{1} m\left(N-\alpha_{1} m-2\right)\right)^{q /(p q-m n)}\left(\alpha_{2} n\left(N-\alpha_{2} n-2\right)\right)^{m /(p q-m n)} .
\end{aligned}
$$

Let

$$
U(x)=M_{1}\left(1+|x|^{2}\right)^{-\alpha_{1} / 2}, \quad V(x)=M_{2}\left(1+|x|^{2}\right)^{-\alpha_{2} / 2}, \quad x \in D^{c},
$$

where

$$
\alpha_{1}=\frac{2(p+n)}{p q-m n}, \quad \alpha_{2}=\frac{2(q+m)}{p q-m n} .
$$


Then $(U, V)$ satisfy

$$
\left\{\begin{aligned}
-\Delta U^{m}-V^{p} & =M_{1}^{m} \alpha_{1} m\left(\alpha_{1} m+2\right)\left(1+|x|^{2}\right)^{-m \alpha_{1} / 2-2}, & & x \in D^{c}, \\
-\Delta V^{n}-U^{q} & =M_{2}^{n} \alpha_{2} n\left(\alpha_{2} n+2\right)\left(1+|x|^{2}\right)^{-n \alpha_{2} / 2-2}, & & x \in D^{c} .
\end{aligned}\right.
$$

Now for $f_{1}(x), f_{2}(x), u_{0}(x), v_{0}(x)$ small enough such that

$$
f_{1}(x) \leq \frac{\partial U^{m}}{\partial \nu}, \quad f_{2}(x) \leq \frac{\partial V^{n}}{\partial \nu}, \quad x \in \partial D,
$$

and

$$
u_{0}(x) \leq U(x), v_{0}(x) \leq V(x), \quad x \in D^{c},
$$

$(U(x), V(x))$ is a global supersolution of (1.1) by Lemma 1.

\section{References}

[1] M. Aripov. Method of the Standard Equation for the Solution of the Nonlinear Value Problem. Fan, Tashkent, 1988.

[2] M. Aripov and J. Muhammadiev. Asymptotic behaviour of automodel solutions for one system of quasilinear equations of parabolic type. Buletin StiintificUniversitatea din Pitesti, Seria Matematica si Informatica, 3:19-40, 1999.

[3] M. Aripov and S. Sadullaeva. Qualitative properties of solutions of a doubly nonlinear reaction-diffusion system with a source. Journal of Applied Mathematics and Physics, 3(9):1090-1099, 2015. http://dx.doi.org/10.4236/jamp.2015.39135.

[4] G. Boyadjiev and N. Kutev. Diffraction problems for quasilinear reaction-diffusion systems. Nonlinear Analysis, 55(7-8):905-926, 2003. http://dx.doi.org/10.1016/j.na.2003.08.006.

[5] M. Escobedo and M.A. Herrero. Boundedness and blow up for a semilinear reaction-diffusion system. Journal of Differential Equations, 89(1):176-202, 1991. http://dx.doi.org/10.1016/0022-0396(91)90118-S.

[6] H. Fujita. On the blowing up of solutions of the Cauchy problem for $u_{t}=$ $\delta u+u^{\alpha+1}$. Journal of the Faculty of Science, University of Tokyo. Sect. 1, Mathematics, astronomy, physics, chemistry, 13:109-124, 1966.

[7] K. Hayakawa. On nonexistence of global solutions of some semilinear parabolic differential equations. Proceedings of the Japan Academy, 49(7):503-505, 1973. http://dx.doi.org/10.3792/pja/1195519254.

[8] P. Lei and S. Zheng. Global and nonglobal weak solutions to a degenerate parabolic system. Journal of Mathematical Analysis and Applications, 324(1):177-198, 2006. http://dx.doi.org/10.1016/j.jmaa.2005.12.012.

[9] A.A. Samarskii, V.A. Galaktionov, S.P. Kurdyumov and A.P. Mikhailov. Blowup in quasilinear parabolic equations, volume 19 of De Gruyter Expositions in Mathematics. Walter de Gruyter, Berlin, 1995.

[10] F.B. Weissler. Existence and non-existence of global solutions for a semilinear heat equation. Israel Journal of Mathematics, 38(1-2):29-40, 1981. http://dx.doi.org/10.1007/BF02761845. 
[11] J. Yang, S. Zheng and Ch. Qu. Fujita phenomenon in inhomogeneous fast diffusion system. Zeitschrift für angewandte Mathematik und Physik, 64(2):311-319, 2012. http://dx.doi.org/10.1007/s00033-012-0239-3.

[12] X. Zeng. The critical exponents for the quasi-linear parabolic equations with inhomogeneous terms. Journal of Mathematical Analysis and Applications, 332(2):1408-1424, 2007. http://dx.doi.org/10.1016/j.jmaa.2006.11.034.

[13] S. Zheng and W. Wang. Critical exponents for a nonlinear diffusion system. Nonlinear Analysis: Theory, Methods \& Applications, 67(4):1190-1210, 2007. http://dx.doi.org/10.1016/j.na.2006.07.007. 